\title{
Dynamics of Bianchi I Universe in Extended Gravity with Scale Factors
}

\author{
Fakhereh MD. Esmaeili \\ School of Physics, University of Hyderabad, Hyderabad, India \\ Email: astrosat92@gmail.com
}

How to cite this paper: Esmaeili, F.MD. (2018) Dynamics of Bianchi I Universe in Extended Gravity with Scale Factors. Journal of High Energy Physics, Gravitation and Cosmology, 4, 716-730.

https://doi.org/10.4236/jhepgc.2018.44040

Received: July 17, 2018

Accepted: October 13, 2018

Published: October 16, 2018

Copyright $\odot 2018$ by author and Scientific Research Publishing Inc. This work is licensed under the Creative Commons Attribution International License (CC BY 4.0).

http://creativecommons.org/licenses/by/4.0/

(c) (i) Open Access

\begin{abstract}
In this paper, the dynamical behavior of an anisotropic universe in an extended gravity e.g. the $f(R, T)$ theory of gravity is studied. We use $f(R, T)=R+2 \mu T$, where $R$ is the Ricci scalar, $T$ is the trace of energy-momentum tensor and $\mu$ is a constant. Two cosmological models are constructed using the power law expansion and hybrid law cosmology in $\mathrm{Bi}-$ anchi type I universe, where the matter field is considered to be a perfect fluid. It is found that in both the cases the anisotropic behavior is in agreement with the observational results. The state finder diagnostic pair and energy conditions are also calculated and analyzed.
\end{abstract}

\section{Keywords}

$f(R, T)$ Gravity, Bianchi Type I, Perfect Fluid, Anisotropic Universe, SEC

\section{Introduction}

Einstein's General Theory of Relativity (GR) is one of the revolutionary concepts in modern physics. This equation results out of the outstanding interplay between matter and space-time. Based on Einstein equation $G_{\mu \nu}-\frac{1}{2} g_{\mu \nu} R=\kappa T_{i j}$, the presence of energy-momentum distribution has shown how gravity influenced space-time and how the curvature of space-time acts on gravity simultaneously. While (GR) is successful in several physical aspects, some astrophysical and cosmological issues still have not found their appropriate explanation by GR. Late time cosmic acceleration, is the most significant result that is indescribable in theory of GR. Of the proposed alternatives of the general theory of gravity, modified gravity theory is found to be one of the more appealing solutions. The recent cosmological observations of Type Ia supernovae 
(SN Ia) [1] [2] [3], the cosmic ray Microwave Background (CMB) [4] [5], Wilkinson Microwave Planck and the Sloan Digital Sky Survey [6] [7] strongly suggest the acceleration of the universe.

An unprecedented view of our dark universe has been shown by the recent discovery of the gravitational wave by LIGO (Laser Interferometer GravitationalWave Observatory). With the help of precise measurement of advanced LIGO, we will be able to test the differences between Einstein's General Theory of Relativity and it's extended theories [8] [9].

Primary ideas of generalization of the GR have gone beyond the Einstein-Hilbert action. The generalization of interest here is including the term of $f(R)$ theory of gravity in the Einstein-Hilbert action. The presence of a late time cosmic acceleration of the universe can indeed be explained by $f(R)$ gravity [10]. Later on, Harko et al. [11] proposed some types of that $f(R, T)$ terms that can be included in Einstein-Hilbert action to describe the late time cosmology. After an essential suggestion of changing the action, there have been many other suggestions put forth in recent times that address critical issues in cosmology. A specific example is the $f\left(R, L_{m}\right)$ gravity [12], which considered a relativistically co-variant model of interacting dark energy, based on the principle of the least action. Several cosmological models with different aspects have been constructed and analyzed in the last decade. Perlmutter et al. [13] has found the first evidence for the positive-cosmological-constant universe that shows expansion of universe based on observations of a set of 40 high-red-shift supernovae.

Bamba [14] has gone through different approaches of testing dark energy and alternative models of extended gravity by cosmography. An approach to work on this problem is to modify the regular part of the Einstein field equation where the standard Einstein-Hilbert action replaced by an arbitrary function of Ricci scalar $R$ [15]. Yousaf [16] has discussed irregular energy density in the presence of imperfect fluid for $f(R, T)$. Alves et al. [17] considered the extra polarization modes and found corresponding gravitational waves in $f(R, T)$ of the model. Zaregonbadi [18] solved the dark matter in galaxy scale in $f(R, T)$ gravity. Mishra et al. [19] obtained transition from early deceleration to late-time cosmic acceleration due to different deceleration parameter in the presence of hybrid scale factor.

Most studies on $f(R, T)$ gravity are with isotropic universes. However, Mishra et al. [20] [21] have studied the dynamical behavior of the anisotropic cosmological models in detail. A universe filled by perfect fluid in cylindrically symmetric space time in two classes of $f(R, T)$ gravity has been studied in [22]. In this respect, Halife [23] studied the anisotropic Bianchi type I universe in the presence of quark matter source in $f(R, T)$ with cosmological constant. Naser [24] added new scale factor as $R=\sinh ^{\frac{1}{n}}(\beta t)$ in Bianchi Type-V Cosmology in $f(R, T)$ gravity. Several cosmological models were analysed on the perspective aspect of the anisotropic universe by considering recent 
observation of supernovae type I as a perfect standard candle and studied their spectrum [25] [26]. Harko et al. [11] considered three different classes of $f(R, T)$ gravity such as 1) $f(R, T)=R+2 f(T), 2) \quad f(R, T)=f_{1}(R)+f_{2}(T)$ and 3) $f(R, T)=f_{1}(R)+f_{2}(R) f_{3}(T)$.

Simultaneously [27] investigated the role of cosmological constant to obtain the proper solution for relativistic stars by used of anisotropic fluid structure of the cosmic stellar filament. There is a provoking discussion in [28], wherein the Lemaitre- Tolman- Bondi (LTB) space-time is applied as a powerful tool in describing the cosmic gravitational collapse. Hence it was suggested to look for the possibility of a viable polynomial model of $f(R)$ gravity for collapsing of an inhomogenous LTB space-time.

The arbitrariness in the choice of different functional forms of $f(R, T)$ to the need for constraints. Some constraints on $f(R)$ gravity from the energy conditions have been considered by Santos et al. [29]. In this direction, considerable research has been carried out in $f(R, T)$ gravity to find the emergence of relativistic compact stellar objects [30] and their stability in the background of some observations. The energy density has remained positive and approached to the maximum value at the core of stars. Yousaf et al. [31] have discussed the effect of $f(R, T)$ gravity on formation of spherically symmetric wormhole under distinct distribution of matter content as perfect, barotropic and anisotropic fluid only by using the validity of WEC (weak energy condition) and NEC(null energy condition). It is worth setting up the energy conditions of field equations in order to constraint the energy-momentum tensor selection in $f(R, T)$ gravity in an anisotropic universe, which is what this paper attempts to do. In the case all the energy conditions are satisfied then we can refer to $T_{\mu \nu}$ as the true sources of energy and momentum. Santos et. al. The study of dark energy is possible through its equation of state parameter $\omega=\frac{p}{\rho}$. If the value of $\omega=-1$ this is equivalent to the cosmological constant $\Lambda$. For minimally coupled scalar field such as quintessence $\left(-1 \leq \omega \leq \frac{-1}{3}\right)$ and phantom $(\omega \leq-1)$. From results of supernovae data CMBR anisotopy, the values are $-1.67 \leq \omega \leq-0.62$ and $-1.33 \leq \omega \leq-0.79$ respectively.The notion of energy conditions have been used to explore viable models of gravity in [32]. The viable solutions are explained by assuming Starobinsky model $f(R, T)=R+R^{2}+T$ and specific shape function.

It is to be noted that perfect fluid is one of the matter of the universe which go through variation and contraction of the action. At the back drop of our mind, we would be interested to see that $f(R, T)$ would gradually be fitted into GR equation. The present paper is arranged as follows. In Section II, we present the field equations obtained from the substitution of the Bianchi type I space-time metric in the $f(R, T)$ gravity. Some cosmological parameters of this approach are also defined. In Section III, we have constructed the cosmological models with the power law cosmology and hybrid cosmology. The physical behaviors of 
the models along with the energy conditions are described in Section IV. Concluding remarks are given in Section V.

\section{Basic Equations}

In this section, we will derive the field equations of $f(R, T)$ gravity in the framework of Bianchi type I space-time and energy momentum tensor in the form of perfect fluid with the choice of the functional $f(R, T)=R+2 \mu T$, where $\mu$ is an arbitrary constant.The action in the $f(R, T)$ theory of gravity can be written as .

$$
S=\int\left(\frac{1}{16 \pi} f(R, T)+\mathcal{L}_{m}\right) \sqrt{-g} \mathrm{~d}^{4} x
$$

with $f(R, T)$ be a function of $R$ and $T, g$ is the determinant of the metric and $\mathcal{L}_{m}$ is the matter Lagrangian density. The energy-momentum tensor can be defined as $T_{i j}=-\frac{2}{\sqrt{-g}} \frac{\delta\left(\sqrt{-} g \mathcal{L}_{m}\right)}{\delta g^{i j}}$. It is to be mentioned that here the matter Lagrangian is assumed to be dependent only on the metric tensor $g_{i j}$ and not on its derivatives. So, the stress-energy tensor can be expressed as :

$$
T_{i j}=g_{i j} \mathcal{L}_{m}-\frac{\delta \mathcal{L}_{m}}{\delta g^{i j}} .
$$

Now, varying action (1) with respect to the metric component $g_{i j}$, the field equations of $f(R, T)$ gravity can be obtained as

$$
\begin{aligned}
& f(R, T) R_{i j}-\frac{1}{2} f(R, T) g_{i j}+\left(g_{i j} \square-\nabla_{i} \nabla_{j}\right) f_{R}(R, T) \\
& =8 \pi T_{i j}-f_{T}(R, T) T_{i j}-f_{T}(R, T) \Theta_{i j},
\end{aligned}
$$

with $R_{i j}$ being the Ricci tensor, where $\Theta_{i j}=-2 T_{i j}+g_{i j} \mathcal{L}_{m}-2 g^{\alpha \beta} \frac{\partial^{2} \mathcal{L}_{m}}{\partial g^{i j} \partial g^{\alpha \beta}}$. Here, $f_{R}=\frac{\partial}{\partial R}, \quad f_{T}=\frac{\partial}{\partial T}$ and $\square \equiv \nabla^{i} \nabla_{j}$, with $\nabla_{i}$ being the covariant derivative.

We consider the homogeneous and anisotropic Bianchi universe as

$$
\mathrm{d} s^{2}=\mathrm{d} t^{2}-b_{1}^{2} \mathrm{~d} x^{2}-b_{2}^{2}\left(\mathrm{~d} y^{2}+\mathrm{d} z^{2}\right)
$$

where the metric potentials $b_{1}$ and $b_{2}$ are function of the cosmic time. The energy-momentum tensor is

$$
T_{i j}=(\rho+p) u_{i} u_{j}-p g_{i j} .
$$

Here, $\rho$ and $p$, respectively, denote the matter-energy density and proper pressure of the matter field and $u^{i}=(0,0,0,1)$ is the four-velocity vector of the fluid in a co-moving coordinate system that satisfies $u^{i} u_{i}=1$. Now, by assuming the matter Lagrangian as $\mathcal{L}_{m}=-p$, the field equations Equation (3) for the choice $f(R, T)=R+2 \mu T$ can be reduced to

$$
R_{i j}-\frac{1}{2} R g_{i j}=(8 \pi+2 \mu) T_{i j}+\Lambda g_{i j} \text {. }
$$


where, $\Lambda=(\rho-p) \mu$. In the co-moving coordinate system, the field equations are

$$
\begin{gathered}
2 \frac{\ddot{b}_{2}}{b_{2}}+\frac{\dot{b}_{2}^{2}}{b_{2}^{2}}=-(8 \pi+3 \mu) p+\rho \mu, \\
\frac{\dot{b}_{1} \dot{b}_{2}}{b_{1} b_{2}}+\frac{\ddot{b_{2}}}{b_{2}}+\frac{\ddot{b_{1}}}{b_{1}}=-(8 \pi+3 \mu) p+\rho \mu, \\
2 \frac{\dot{b}_{1} \dot{b}_{2}}{b_{1} b_{2}}+\frac{\dot{b}_{2}^{2}}{b_{1}^{2}}=(8 \pi+3 \mu) \rho-p \mu,
\end{gathered}
$$

An over dot on the field variables represent the ordinary derivative with respect to the cosmic time. In order to understand the model physically, we will consider the directional Hubble rates in the form $H_{x}=\frac{\dot{b_{1}}}{b_{1}}$ and $H_{y}=H_{z}=\frac{\dot{b_{2}}}{b_{2}}$, which subsequently yield the mean Hubble parameter as $H=\frac{\dot{a}}{a}=\frac{1}{3}\left(H_{x}+2 H_{y}\right)$, with (a) denotes the mean scale factor of the universe. So, the set of field Equations (7)-(9) can be expressed in term of the scale factor (a) as:

$$
\begin{gathered}
\frac{3}{k+2}\left[2 \frac{\ddot{a}}{a}+\left(\frac{5-2 k}{k+2}\right)\left(\frac{\dot{a}}{a}\right)^{2}\right]=\mu \rho-\beta p \\
\frac{3}{k+2}\left[(k+1) \frac{\ddot{a}}{a}+\left(\frac{2 k^{2}+1}{k+2}\right)\left(\frac{\dot{a}}{a}\right)^{2}\right] \mu \rho-\beta p \\
\frac{9(2 k+1)}{(k+2)^{2}}\left(\frac{\dot{a}}{a}\right)^{2}=\beta \rho-\mu p,
\end{gathered}
$$

where we have defined $\beta \equiv 8 \pi+3 \mu$. By performing algebraic manipulation on Equations (10)-(12), the pressure $(p)$ and energy density $(\rho)$ of the model concerning the scale factor can be expressed respectively as

$$
p=\left(\frac{6 \beta}{k+2}\right) \frac{\ddot{a}}{a}+\frac{9}{(k+2)^{2}}\left[3 \beta-\mu-\frac{2}{3} \beta(k+2)-2 k \mu\right]\left(\frac{\dot{a}}{a}\right)^{2}
$$

and

$$
\rho=\left(\frac{6 \beta}{k+2}\right) \frac{\ddot{a}}{a}+\frac{9}{(k+2)^{2}}\left[3 \mu-\beta-\frac{2}{3} \mu(k+2)-2 k \beta\right]\left(\frac{\dot{a}}{a}\right)^{2}
$$

Subsequently, with respect to the scale factor, the equation of state (EoS) parameter $\omega$ is obtained as

$$
\omega=\frac{p}{\rho}=\frac{\left(\frac{6 \beta}{k+2}\right) \frac{\ddot{a}}{a}+\frac{9}{(k+2)^{2}}\left[3 \beta-\mu-\frac{2}{3} \beta(k+2)-2 k \mu\right]\left(\frac{\dot{a}}{a}\right)^{2}}{\left(\frac{6 \beta}{k+2}\right) \frac{\ddot{a}}{a}+\frac{9}{(k+2)^{2}}\left[3 \mu-\beta-\frac{2}{3} \mu(k+2)-2 k \beta\right]\left(\frac{\dot{a}}{a}\right)^{2}}
$$

We also define an effective cosmological constant (ECC) for the model as

$$
\Lambda=\frac{2 \mu}{\mu+\beta}\left[\left(\frac{3}{k+2}\right) \frac{\ddot{a}}{a}+\left(\frac{\dot{a}}{a}\right)^{2} \frac{6}{k+2}\right]
$$




\section{Cosmological Features of the Model}

In this section, we will discuss the cosmological features of the model with the scale factor being in the form of the power law and hybrid law cosmologies.

\subsection{Power Law Cosmology}

In the previous section, we have expressed the physical parameters of the model with respect to the Hubble parameter. In order to study the dynamics of the universe, we need to know the parameters with respect to the cosmic time, hence we consider here the power law function in the form $a=t^{\frac{m}{3}}$. This type of assumption is quite helpful to understand the background cosmology, which can also be checked in the solutions for the standard cosmology Friedman equations. In order to employ some amount of anisotropic, we have assumed here $H_{x}=k H_{y}$, where $(k)$ is a constant. It can be seen that when $k=1$, the space time reduces to standard FRW model. With these assumptions, we obtain the directional and mean Hubble parameters, respectively, as $H_{x}=\frac{m k}{(k+2) t}$, $H_{y}=H_{z}=\frac{m}{(k+2) t}$ and $H=\frac{1}{3}\left(H_{x}+2 H_{y}\right)=\frac{m}{3 t}$. So, the pressure and energy density can be obtained as

$$
\begin{gathered}
p=\frac{m(4(4+2 k-3 m) \pi+(6-4 m+k(3+m)) \mu)}{4(2+k)^{2} t^{2}(2 \pi+\mu)(4 \pi+\mu)} \\
\rho=\frac{-2 m \mu(k+2)+m^{2}(-8 \pi-2 k(8 \pi+3 \mu))}{(2+k)^{2} t^{2}\left(\mu^{2}-(8 \pi+3 \mu)^{2}\right)}
\end{gathered}
$$

The graphical behavior of the pressure and energy density have been represented in Figure 1 and Figure 2 respectively. The pressure remains entirely in the negative domain. It starts increasing at initial phase and after a specific time period, it remains constant; however it never touches the horizontal axis. The behavior of the variation of pressure with respect to cosmic time are

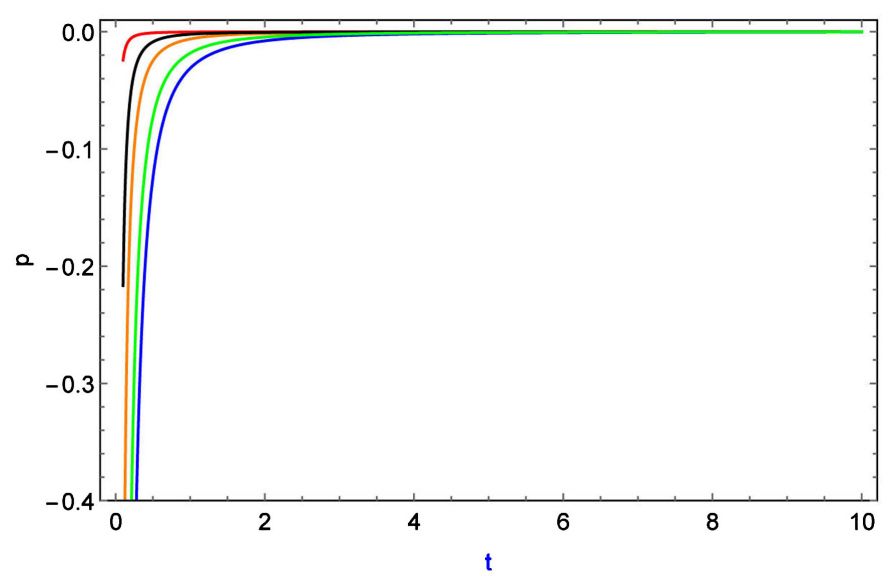

Figure 1. Plot of pressure $(p)$ versus cosmic time for power law. 


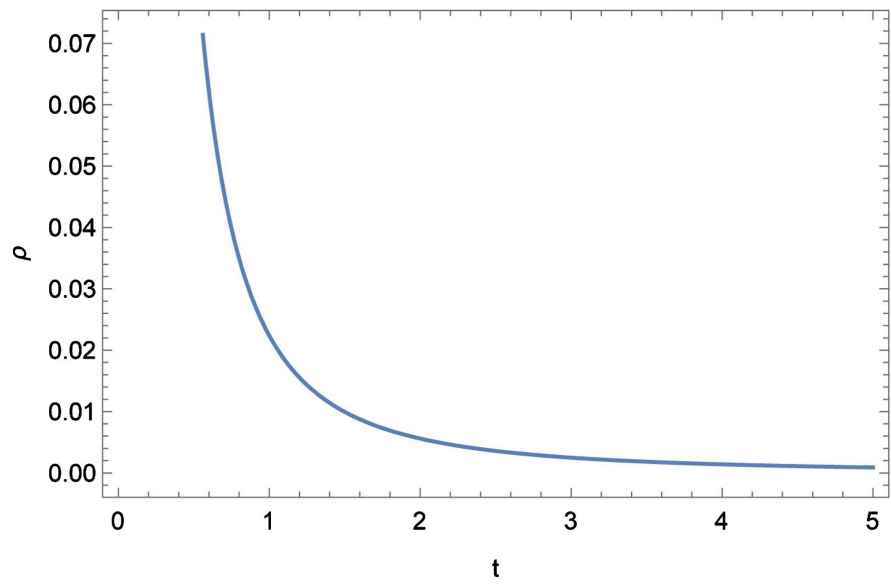

Figure 2. Plot of energy density $(\rho)$ versus cosmic time for power law.

shown for $k=0.12$ and various range of $m: 1.42,1.5,1.6,1.8,2$ and $\mu: 0.01$, $1.15,1.8,0.07,0.001$ as anisotropy parameter and scaling constant where have shown with Red, Black, Orange, Green and Blue respectively. Pressure minimized on $m>\frac{8 \pi+3 \mu}{6 \pi+2 \mu}$ and $0<k<\frac{-16 \pi+12 m \pi-6 \mu+4 m \mu}{8 \pi+3 \mu+m \mu}$.

Subsequently the EoS parameter and ECC can be obtained as

$$
\begin{gathered}
\omega=\frac{4 \pi(4+2 k-3 m)+\mu(6-4 m+k(3+m))}{4 m \pi(1+2 k+(2+k+3 k m) \mu} \\
\Lambda=\frac{\mu m(m-1)}{2 t^{2}(2+k)(2 \pi+\mu)}
\end{gathered}
$$

In Figure 3 and Figure 4, we have respectively graphically represented the EoS parameter and ECC. As it is provided in Figure 3, the EOS parameter is considered and represented as increasing function of time whereas the primary points of evolutionary process starts from 0.08 while it incrementally shifting to -0.6 while it remains on negative domain. It can be seen that the EoS parameter strongly depends on the anisotropic parameter $k$.

In Figure 4, ECC depend on the cosmic time $(t)$. A function which decreased by time and approaches to its small positive value at present epoch. In the early universe, $(\mu)$ takes a very large value and after that decreasing as time increases. Therefore it explained that the energy density is predominance as a decreasing functional behavior in positive domain permanently.

\subsection{Hybrid Scale Factor}

The power-law and exponential law cosmologies are having constant deceleration parameter and only can be used to describe the epoch based evolution of the universe. These cosmologies do not exhibit the transition of the universe from deceleration to acceleration. So, in this section, in order to show the early deceleration and late time acceleration, we have considered a scale factor in the form $a=\mathrm{e}^{\frac{\gamma}{3} t} t^{\frac{\alpha}{3}}$ known as hybrid scale factor [19] [21] [33] [34]. 


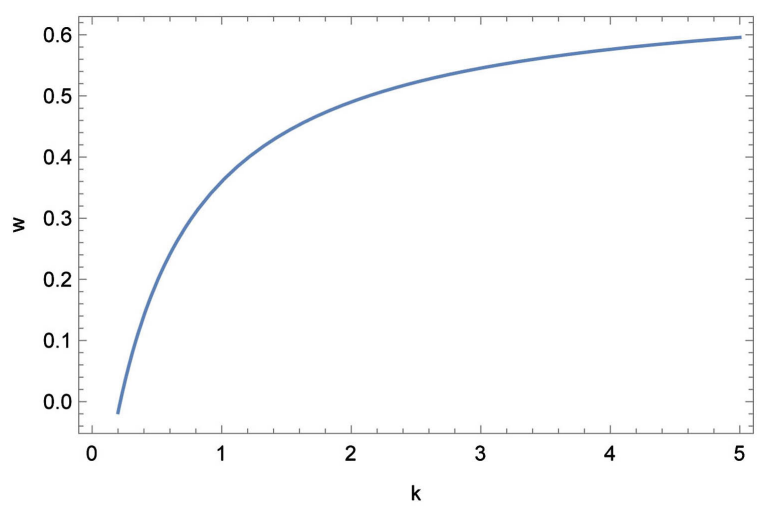

Figure 3. Plot of EoS parameter $(\omega)$ versus $k$ for power law.

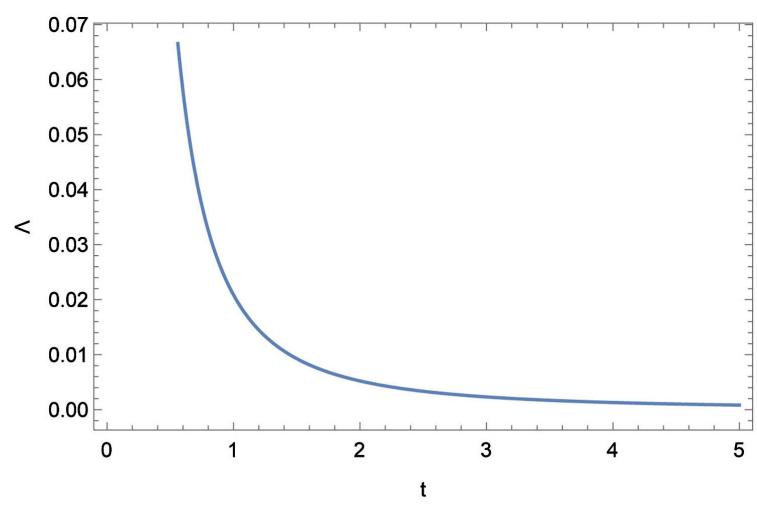

Figure 4. Plot of ECC $(\Lambda)$ versus cosmic time for power law.

This scale factor is having two components: one component behavior is like exponential universe and the other is the power law expansion. Moreover, in this scale factor the exponential component dominates at late phase while in the early phase of cosmic evolution the power law component dominates. It can be noted that when $\gamma=0$, the scale factor reduces to a power law expansion whereas when for $\alpha=0$, only the exponential law can be recovered. For this scale factor, the Hubble rate is $H(t)=\frac{\alpha}{3 t}+\frac{\gamma}{3}, H_{x}=\frac{k}{k+2}\left(\gamma+\frac{\alpha}{t}\right)$, $H_{y}=H_{z}=\frac{1}{k+2}\left(\gamma+\frac{\alpha}{t}\right)$. The pressure and energy density for the hybrid scale factor can be respectively obtained as:

$$
\begin{gathered}
p=\frac{-2(2+k) \alpha \beta+(\alpha+t \gamma)^{2}(3 \beta-\mu)-2 k(\alpha+t \gamma)^{2} \mu}{(2+k)^{2} t^{2}\left(-\beta^{2}+\mu^{2}\right)} \\
\rho=-\frac{(2 k \beta+\beta-3 \mu)(\alpha+t \gamma)^{2}+2(2+k) \alpha \mu}{(2+k)^{2} t^{2}\left(-\beta^{2}+\mu^{2}\right)}
\end{gathered}
$$

In Figure 5 and Figure 6 we observe the fluid pressure as a function of time and variation of energy density. In the Figure 5 we assume representative value of the anisotropy i.e. $k=0.25$, for $\alpha=0.1$ In order to minimizing the pressure it is required to inequality of 


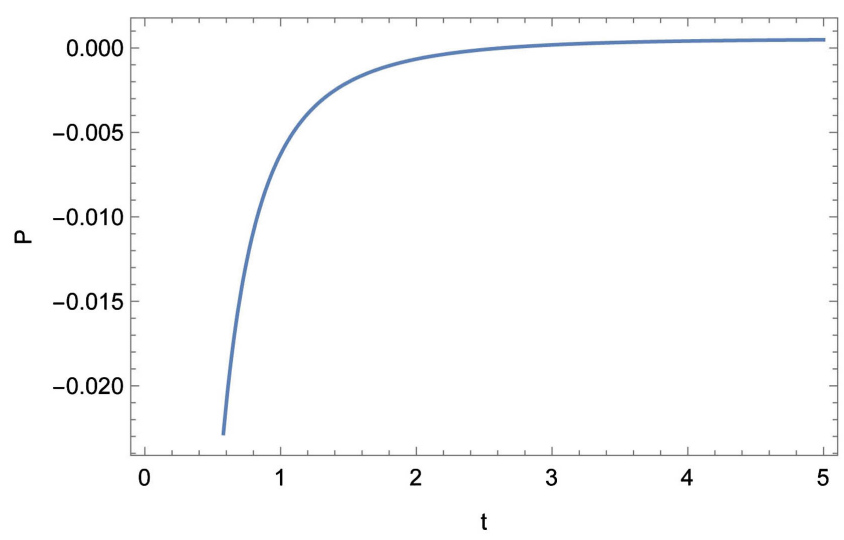

Figure 5. Plot of pressure $(p)$ versus cosmic time for hybrid scale factor.

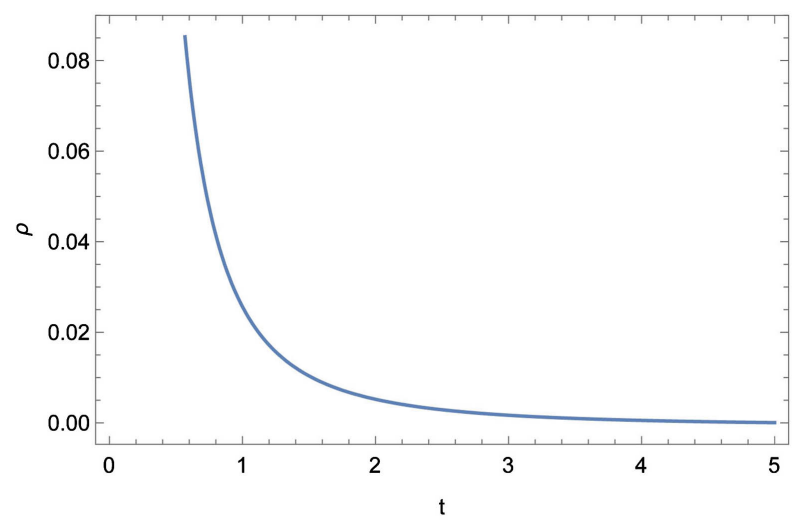

Figure 6. Plot of energy density $(\rho)$ versus cosmic time for hybrid scale factor.

$$
\frac{-16 \pi+12 \pi \beta+6 \mu+4 \beta \mu}{8 \pi+3 \mu+\beta \mu}<k<\frac{12 \pi+4 \mu}{\mu}
$$

the pressure raised up to its lowest negative value.

From Figure 6 we observe that the energy density always remain in the positive domain and it has decreased to its small value at late times. At the same time the energy density start decreasing from a high value and decreases with increase in the time period. On the other hand, the pressure raised up to its lowest negative value from some large negative value $(\approx 0.023)$ and gradually vanishing to $\omega \rightarrow 0$ at late times. We have obtained the EoS parameter $\omega$ and effective cosmological constant $\Lambda$ can be obtained as

$$
\begin{gathered}
w=-\frac{-2(2+k) \alpha \beta+(\alpha+t \gamma)^{2}(3 \beta-\mu)-2 k(\alpha+t \gamma)^{2} \mu}{(2 k \beta+\beta-3 \mu)(\alpha+t \gamma)^{2}+2(2+k) \alpha \mu} \\
\Lambda=\frac{2\left((-1+\alpha) \alpha+2 t \gamma \alpha+t^{2} \gamma^{2}\right) \mu}{(2+k) t^{2}(\alpha+\mu)}
\end{gathered}
$$

Figure 7 represents evolution of EoS with respect to cosmic time. In the present study for hybrid case $\omega$ remains in the negative domain favoring a quintessence phase. in the present epoch $\omega$ get the lowest value of -0.32 corresponding to $k=0.25$. 


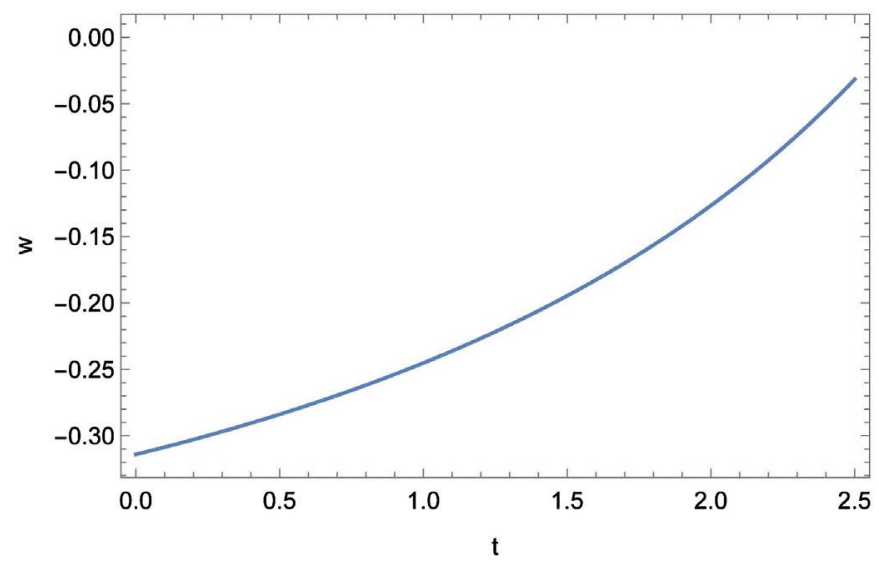

Figure 7. Plot of EoS parameter $(\omega)$ versus cosmic time for hybrid scale factor.

\section{Physical Behavior of the Models}

The scalar expansion and deceleration parameter of the model can be obtained as $\theta=3 H(t)=\frac{m}{t}$ and $q=-1+\frac{3}{m}$. We can infer that the scalar expansion vanishes at late time and deceleration parameter remains constant at -1 . It can be noted that when $m>3$, the model decelerates and accelerates for $m<3$. The volume scale factor $V$ and the ratio of anisotropy $\mathcal{A}=\frac{1}{3} \Sigma\left(\frac{\Delta H_{i}}{H}\right)^{2}$ can be respectively calculated as $t^{m}$ and $\frac{2(k-1)^{2}}{(k+2)^{2}}$. As usual for $k=1$, the model reduces to an isotropic universe. The state finder diagnostic pair which give some insight to the geometrical part of the model. The pair for the power law cosmology can be obtained as $r=\frac{(m-3)(m-6)}{m^{2}}, s=\frac{r-1}{3(q-1 / 2)}=\frac{2}{m}$.

For hybrid cosmology $\theta=\frac{\beta}{t}+\gamma$ and $q=-1+\frac{3 \beta}{(\beta+\gamma t * \gamma)^{2}}$. Figure 8 has shown evolution of deceleration parameter in terms of cosmic time for transition from deceleration to acceleration phase. In this model we have positive values of $q$ in the range of $\frac{3 \beta}{\beta+\gamma t}>1$ for decelerated universe and facing to negative values of $q$ for accelerated universe at the late time for $\frac{3 \beta}{\beta+\gamma t} \leq 1$. Similarly for a hybrid cosmology, the pair can be obtained as

$$
r=1-\frac{9 \alpha(-2+\alpha+t \gamma)}{(\alpha+\gamma t)^{3}}, \text { and } s=\frac{r-1}{3\left(q-\left(\frac{1}{2}\right)\right)}=-\frac{3 \alpha(-2+\alpha-t \gamma)}{(\alpha+t \gamma)^{3}\left(-\frac{3}{2}+\frac{3 \alpha}{(\alpha+t \gamma)^{2}}\right)}
$$

Energy conditions as a sets of linear equations put some additional constraints on the model. However Equation (25) has demonstrated that the field equation has been physically approved. Energy conditions derived as: 


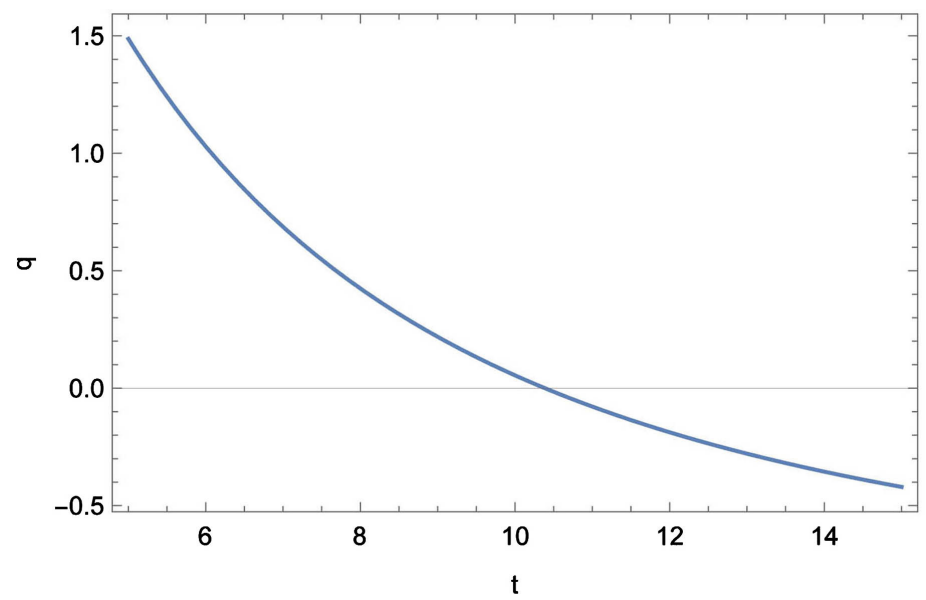

Figure 8. Plot of Deceleration parameter $(\omega)$ versus cosmic time for hybrid scale factor.

$$
\left\{\begin{array}{l}
\text { Null Energy Conditions(NEC): } \rho+p \geq 0 \\
\text { Weak Energy Condition( (WEC) }: \rho+p \geq 0, \rho \geq 0 \\
\text { Strong Energy Condition(SEC): } \rho+3 p \geq 0, \rho+p \geq 0 \\
\text { Dominant Energy Condition(DEC): } \rho \pm p \geq 0, \rho \geq 0
\end{array}\right.
$$

Now, the energy conditions for power law cosmology can be obtained as:

$$
\begin{gathered}
\rho+p=\frac{2 m(2+k+(k-1) m)}{(2+k)^{2} t^{2}(\beta-\mu)} \\
\rho+3 p=\frac{2 m((6-4 m+k(m+3)) \beta+(2+k+3 m k) \mu)}{(2+k)^{2} t^{2}\left(\beta^{2}-\mu^{2}\right)} \\
\rho-p=\frac{2 m(m-1)}{(2+k) t^{2}(\beta+\mu)}
\end{gathered}
$$

In Figure 9, corresponding graphical behavior of energy conditions for power law cosmology were plotted for $k=0.601$ and various values of $m$. In the present model all the energy conditions have been satisfied. The physical trend of all cases have remained almost the same for various choice of scaling constant $\mu$.

Similarly, for a hybrid cosmology, the energy conditions for the model can be calculated

$$
\rho+p=\frac{2 \alpha(2+k+(-1+k) \alpha)+4(-1+k) t \alpha \gamma+2(-1+k) t^{2} \gamma^{2}}{(2+k)^{2} t^{2}(\beta-\mu)}
$$

$$
\begin{gathered}
3 p+\rho=\frac{2\left(\alpha \beta(3(2+k)+2(-4+k) t \gamma)+\alpha(2+k+6 k t \gamma) \mu+\alpha^{2}((-4+k) \beta+3 k \mu)+t^{2} \gamma^{2}((-4+k) \beta+3 k \mu)\right)}{(2+k)^{2} t^{2}\left(\beta^{2}-\mu^{2}\right)} \\
\rho-p=\frac{2\left((-1+\alpha) \alpha+2 t \alpha \gamma+t^{2} \gamma^{2}\right)}{(2+k) t^{2}(\beta+\mu)}
\end{gathered}
$$


Figure 10 shows the graphical behavior of energy conditions for hybrid scale factor for $k=0.25$. It is implied by the $\operatorname{Green}(\mathrm{U})$, Blue(Q) and $\operatorname{Red}(\mathrm{G})$ energy frame which is expressed the dynamical trend for SEC, DEC, WEC. It is clearly shown that all energy conditions have been satisfied. Indeed it is assumed that typically SEC is the only known energy condition which we need to violate in order to find a bounce. An outstanding feature of this models is energy condition satisfaction for some values of the input parameters.

Another notable cases is evaluating the effectiveness of higher curvature on the dynamical variables of spherically compact stars which filled by an imperfect fluid as matter content has been investigated on [35]. One of the well-consistence form of $f(R, T)$ is $f_{1}(R)+f_{2}(R) f_{3}(T)$ which is mostly coped with observational results. The selected non-linear complex model considered as $R+\lambda R^{2} T^{2}$ which has been compared with experiment result s of a neutron star named as $4 \mathrm{U} 1820-30$. Moreover the correspondence Raychaudhuri equations for shear and expansion scalar equations has established for shear and expansion scalar.

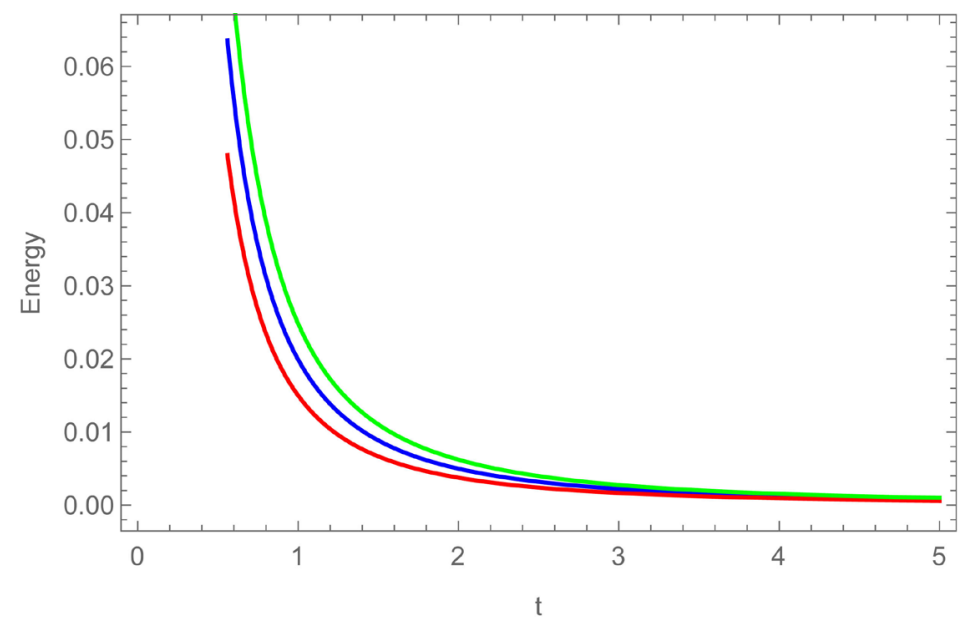

Figure 9. Plot of energy conditions versus cosmic time for power law cosmology.

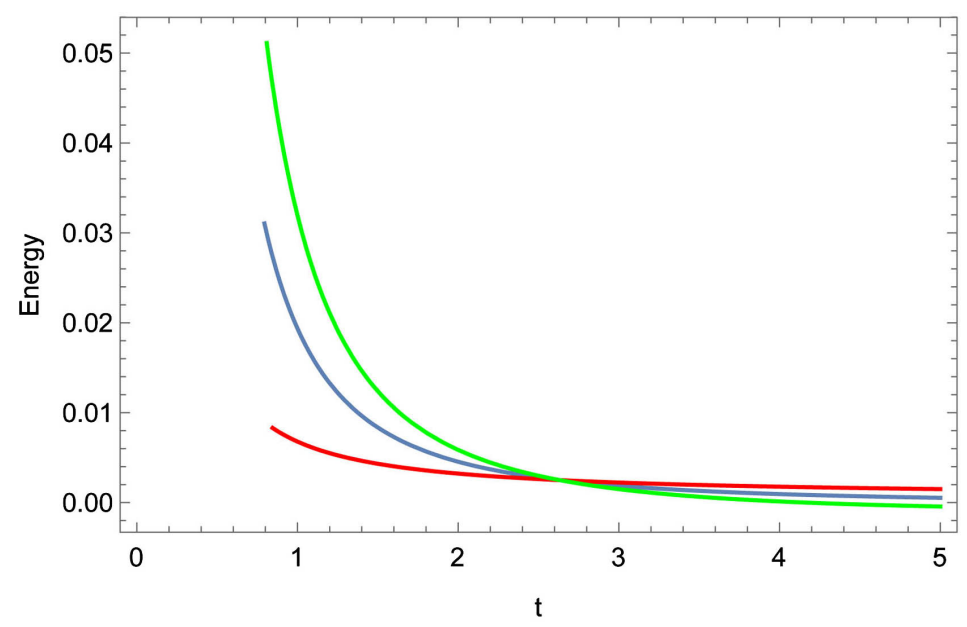

Figure 10. Plot of energy conditions versus cosmic time for hybrid cosmology. 


\section{Conclusion}

The work done in this paper is to investigate the cosmological model obtained in $f(R, T)$ gravity in Bianchi type I space-time with the functional $f(R, T)=R+2 \mu T$. The dynamical parameters are calculated and analyzed with a new mathematical formalism in power law cosmology and hybrid scale factor cosmology. We have shown that there exist various values for inputs parameters which the four energy conditions may be satisfied simultaneously. The state finder diagnostic pair and energy conditions are also studied. The results obtained in both the models are in accordance with the observational findings of accelerated expansion of the universe. Also the result of this work could be extended to a new approach of extended theories of gravity such as $f(R, \square R, T)$ that could lead to unveil the secret of dark energy in modern cosmology. Hence we addressed to some successful studies on this formalism as [36] which lightened the present of a higher derivatives model of $f(R, T)$ by using of energy conditions and evaluate their viability by making some constraints on coefficient of Ricci scalar and energy-momentum tensor. Also for finding more discussions on implication of energy conditions on formation of universe, it is good to present about the Gauss Bonnet invariant model of extended theory of gravity $f(G)$ in [37] which tries to find the constraint on cosmological parameters which essentially is needed for viability of the constructed model.

\section{Acknowledgements}

I would like to thank Professor.Bindu. A. Bambah for her expert advice and encouragement throughout this research paper. The author is thankful to the anonymous referees for constructive comments concerning our manuscript suggestions for the improvement of the paper.

\section{Conflicts of Interest}

The authors declare no conflicts of interest regarding the publication of this paper.

\section{References}

[1] Riess, A.G., et al. (1998) Observational Evidence from Supernovae for an Accelerating Universe and a Cosmological Constant. The Astrophysical Journal, 116, 1009.

[2] Tonry, J.L., et al. (2003) Cosmological Results from High- $z$ Supernovae. The Astrophysical Journal, 594, 1. https://doi.org/10.1086/376865

[3] Komatsu, E., et al. (2009) Five-Year Wilkinson Microwave Anisotropy Probe Observations: Cosmological Interpretation. The Astrophysical Journal Supplement Series, 180, 330. https://doi.org/10.1088/0067-0049/180/2/330

[4] Penzias, A.A. and Wilson, R.W. (1965) A Measurement of Excess Antenna Temperature at $4080 \mathrm{Mc} / \mathrm{s}$. The Astrophysical Journal, 142, 419-421. https://doi.org/10.1086/148307

[5] Dicke, R.H., Peebles, P.J.E., Roll, P.G. and Wilkinson, D.T. (1965) Cosmic 
Black-Body Radiation. The Astrophysical Journal, 142, 414-419. https://doi.org/10.1086/148306

[6] Campbell, H., et al. (2013) Cosmology with Photo-Metrically-Classified Type Ia Supernovae from the SDSS-II Supernova Survey. The Astrophysical Journal, 763, 28. https://doi.org/10.1088/0004-637X/763/2/88

[7] Lampeitl, H. (2009) First-Year Sloan Digital Sky Survey-II (SDSS-II) Supernova Results: Consistency and Constraints with Other Intermediate-Red-Shift Datasets. Monthly Notices of the Royal Astronomical Society, 401, 2331-2342. https://doi.org/10.1111/j.1365-2966.2009.15851.x

[8] Corda, C. (2018) The Future of Gravitational Theories in the Era of the Gravitational Wave Astronomy. International Journal of Modern Physics D, 27, Article ID: 1850060. https://doi.org/10.1142/S0218271818500608

[9] Corda, C. (2009) Interferometric Detection of Gravitational Waves: The Definitive Test for General Relativity. International Journal of Modern Physics D, 18, 2275-2282. https://doi.org/10.1142/S0218271809015904

[10] Sheykhi, A. (2012) Magnetic Strings in $\mathrm{f}(\mathrm{R})$ Gravity. General Relativity and Gravitation, 44, 2271. https://doi.org/10.1007/s10714-012-1388-6

[11] Harko, T., Lobo, F.S.N., Nojiri, S. and Odintsov, S.D. (2011) c Gravity. Physical Review D, 84, 024020. https://doi.org/10.1103/PhysRevD.84.024020

[12] Harko, T. and Lobo, F.S.N. (2010) f (R, $\mathrm{L}_{\mathrm{m}}$ ) Gravity. The European Physical Journal C, 70, 373-379. https://doi.org/10.1140/epjc/s10052-010-1467-3

[13] Perlmutter, S. (1997) Cosmology from Type Ia Supernovae. Bulletin of the American Astronomical Society, 29, 1351.

[14] Bamba, K., Capozziello, S., Nojiri, S. and Odintsov, S.D. (2012) Dark Energy Cosmology: The Equivalent Description via Different Theoretical Models and Cosmography Tests. Astrophysics and Space Science, 342, 155. https://doi.org/10.1007/s10509-012-1181-8

[15] Nojiri, S. and Odintsov, S.D. (2011) Unified Cosmic History in Modified Gravity: From f (R) Theory to Lorentz Non-Invariant Models. Physics Reports, 505, 59. https://doi.org/10.1016/j.physrep.2011.04.001

[16] Yousaf, Z., Bamba, K. and Bhatti, M.Z. (2016) Causes of Irregular Energy Density in f (R, T) Gravity. Physical Review D, 93, Article ID: 124048. https://doi.org/10.1103/PhysRevD.93.124048

[17] Alves, M.E.S., Moraes, P.H.R.S., de Araujo, J.C.N. and Malheiro, M. (2016) Gravitational Waves in $\mathrm{f}(\mathrm{R}, \mathrm{T})$ and $\mathrm{f}\left(\mathrm{R}, \mathrm{T}^{\Phi}\right)$ Theories of Gravity. Physical Review D, 94, Article ID: 024032. https://doi.org/10.1103/PhysRevD.94.024032

[18] Zaregonbadi, R., Farhoudi, M. and Riazi, N. (2016) Dark Matter from f (R, T) Gravity. Physical Review D, 94, Article ID: 084052. https://doi.org/10.1103/PhysRevD.94.084052

[19] Mishra, B. and Tripathy, S.K. (2015) Anisotropic Dark Energy Model with a Hybrid Scale Factor. Modern Physics Letters A, 30, Article ID: 1550175. https://doi.org/10.1142/S0217732315501758

[20] Mishra, B., Tarai, S. and Pacif, S.K.J. (2018) Dynamics of Bianchi VI $\mathrm{h}_{\mathrm{h}}$ Universe with Bulk Viscous Fluid in Modified Gravity. International Journal of Geometric Methods in Modern Physics, 15, Article ID: 1850036. https://doi.org/10.1142/S0219887818500366

[21] Mishra, B., Tarai, S. and Tripathy, S.K. (2018) Cosmological Models with a Hybrid 
Scale Factor in an Extended Gravity Theory. Modern Physics Letters A, 33, Article ID: 1850052. https://doi.org/10.1142/S0217732318500529

[22] Mishra, B. and Vadrevu, S. (2017) Cylindrically Symmetric Cosmological Model of the Universe in Modified Gravity. Astrophysics and Space Science, 362, 26. https://doi.org/10.1007/s10509-017-3006-2

[23] Caglar, H. and Aygn, S. (2017) Bianchi Type-I Universe in $\mathrm{f}(\mathrm{R}, \mathrm{T})$ Modified Gravity with Quark Matter and $\Lambda$. AIP Conference Proceedings, 1815, Article ID: 080008. https://doi.org/10.1063/1.4976440

[24] Ahmed, N. and Pradhan, A. (2014) Bianchi Type-V Cosmology in f (R, T) Gravity with $\Lambda(\mathrm{T})$. International Journal of Theoretical Physics, 53, 289-306. https://doi.org/10.1007/s10773-013-1809-7

[25] Ghodsi, H., Baghram, S. and Habibi, F. (2017) Supernovae Anisotropy Power Spectrum. JCAP, 10, 017.

[26] Chang, Z., Li, X., Lin, H.N., et al. (2014) Constraining Anisotropy of the Universe from Different Groups of Type-Ia Supernovae. The European Physical Journal C, 74, 2821. https://doi.org/10.1140/epjc/s10052-014-2821-7

[27] Yousaf, Z. (2017) Stellar Filaments with Minkowskian Core in the Einstein-Gravity. The European Physical Journal Plus, 132, 276. https://doi.org/10.1140/epjp/i2017-11547-0

[28] Bhatti, M.Z. and Yousaf, Z. (2017) Gravitational Collapse and Dark Universe with LTB Geometry. International Journal of Modern Physics D, 26, Article ID: 1750045.

[29] Santos, J., Alcaniz, J.S., Rebouas, M.J. and Carvalho, F.C. (2007) Energy Conditions in $\mathrm{f}(\mathrm{R})$ Gravity. Physical Review D, 76, Article ID: 083513.

[30] Yousaf, Z., Bhatti, M.Z. and Ilyas, M. (2018) Existence of Compact Structures in $\mathrm{f}$ (R, T) Gravity. The European Physical Journal C, 78, 307. https://doi.org/10.1140/epjc/s10052-018-5797-x

[31] Yousaf, Z., Ilyas, M. and Bhatti, M.Z. (2017) Static Spherical Wormhole Models in $\mathrm{f}$ (R, T) Gravity. The European Physical Journal Plus, 132, 268.

[32] Yousaf, Z. and Bhatti, M.Z. (2018) Some Anisotropic Planar Stellar Models. International Journal of Geometric Methods in Modern Physics, 15, Article ID: 1850160.

[33] Akarsu, O., Kumar, S., Myrzakulov, R., Sami, M. and Xu, L. (2014) Cosmology with Hybrid Expansion Law: Scalar Field Reconstruction of Cosmic History and Observational Constraints. Journal of Cosmology and Astroparticle Physics, 01, 22.

[34] Mishra, B., Tripathy, S.K. and Ray, P.P. (2018) Bianchi-V String Cosmological Model with Dark Energy Anisotropy. Astrophysics and Space Science, 363, 86. https://doi.org/10.1007/s10509-018-3313-2

[35] Bhatti, M.Z., Yousaf, Z. and Ilyas, M. (2017) Evolution of Compact Stars and Dark Dynamical Variables. The European Physical Journal C, 77, 690. https://doi.org/10.1140/epjc/s10052-017-5236-4

[36] Yousaf, Z., Sharif, M., Ilyas, M. and Bhatti, M.Z. (2018) Energy Conditions in Higher Derivative $\mathrm{f}(\mathrm{R}, \mathrm{R}, \mathrm{T})$ Gravity. International Journal of Geometric Methods in Modern Physics, 15, Article ID: 1850146. https://doi.org/10.1142/S0219887818501463

[37] Bamba, K., Ilyas, M., Bhatti, M.Z. and Yousaf, Z. (2017) Energy Conditions in Modified f (G) Gravity. General Relativity and Gravitation, 49, 112. https://doi.org/10.1007/s10714-017-2276-x 